\title{
Mind your weight: 'Motionlessly' sitting between the object and the verb in Japanese
}

\author{
Hing-Yuet Fung*
}

\begin{abstract}
The object in Japanese is often displaced from its canonical position next to the sentence-final verb, due to motivations such as information structure or animacy. Such flexibility allows for an adverb to be placed between the object and the verb. In the literature, there are suggestions for an almost equal preference to place Japanese manner adverbs before or after the object, inferred from both online and offline results. We will present a corpus study with a representative Japanese manner adverb zitto 'motionlessly' to show that either order may be preferred in different accounts of word order variation, but none can satisfy both requirements of distance minimization and accessibility, which are manifested in competing directions in Japanese, a verb-final language. In both accounts, weight has immense effect and should not be neglected. By using two heuristic methods to measure the weight effect, we propose that this case study with an object and an adverb sheds new light on the explanatory power of the distance minimization account, in particular by the Mimimize Domains principle (Hawkins 1994), which operates at both levels of (1) the constituency construction of the full VP, which favors the object-first order, and (2) the Phrasal Combination Domain between the head of object and the verb, which favors the adverb-first order. It is also proposed to implement a complement-and-adjunct distinction in the MiD principle, as a step toward a more effective study method of weight effect which I shall call efficiency profiling.
\end{abstract}

Keywords. performance theory; weight effect; word order; adverb; Japanese; corpus

1. Introduction. Weight effects are often observed on the shifted positions of noun phrases, due to noun modification that adds to the "heaviness" of the constituent, hence the notion heavy NP-shift (e.g. Ross 1967). The phenomenon was once attributed to the Principle of End Weight because English data show that "long, complex phrases tend to come at the ends of clauses" (Wasow 1997; 81). The preference for placing short phrases before long phrases in English (short-before-long preference) has been attributed to a processing preference that tries to minimize the distance between two constituents with lexical or syntactic dependency (Hawkins 1994). End weight in English has been tested on different constructions, often involving the object as a ready target with the verb on the other end of the dependency. Examples (1)(3) are taken from Arnold et al. (2000).

\section{(1) Heavy NP Shift}

a. The waiter brought the wine we had ordered to the table.

b. The waiter brought to the table the wine we had ordered.

\section{(2) Dative Alternation}

* I am very grateful for comments from Stephen Matthews and participants in the 2021 Virtual Annual Meeting of LSA. This article will become part of my dissertation. Abbreviations: MiD=Minimize Domains, $\mathrm{PCD}=\mathrm{Phrasal} \mathrm{Com}-$ bination Domain, $\mathrm{IC}=$ Immediate Constituent, $\mathrm{NPo}=$ accusatively marked noun phrase, $\mathrm{BCCWJ}=\mathrm{Balanced} \mathrm{Corpus}$ of Contemporary Written Japanese. Author: Hing-Yuet Fung, University of Hong Kong (kycs@ connect.hku.hk). 
a. Chris gave a bowl of Mom's traditional cranberry sauce to Terry.

b. Chris gave Terry a bowl of Mom's traditional cranberry sauce.

\section{(3) Verb-Particle}

a. Sandy picked the freshly baked apple pie up.

b. Sandy picked up the freshly baked apple pie.

Examples of shifts include that between the object and a verb particle, though the shift often interacts with other dependencies in the verb phrase domain and the chance to displace the verb particle drops with particles of strong lexical dependency (Lohse et al. 2004). In dative alternations, since the dative phrase is also a noun phrase with variable weight, the final order is predicted from the interaction of multiple factors such as animacy, givenness, and definiteness, in addition to the difference in weight of the two constituents, measured by length in words (Bresnan et al. 2007). In either cases, weight effect is significant but limited in applicability, therefore it is seldom considered to be a part of the core grammatical knowledge of the language being studied. There is a gap in the literature to isolate weight effect on a constituent of minimal interaction with the object and dependency with the verb, for example an adverb.

By the same processing efficiency principle of distance minimization between related constituents, Japanese as a head-final language is shown to possess a long-before-short preference (Yamashita \& Chang 2001). In a ditransitive sentence in Japanese, the object has a canonical position adjacent to the verb, which is in turn strictly placed at the end of the sentence. When the direct object attains certain weight, it shifts ahead of the indirect object and sometimes even ahead of the subject/topic. The indirect object is also able to shift ahead of the subject/ topic with increasing weight but the chance is not as significant as the shift between the direct object and indirect object in cases of heavy direct objects. (4) shows the four possible orders from Yamashita \& Chang (2001).
a. Taro-ga/wa Miki-ni ringo-o ageta.
Taro-NOM/TOP Miki-DAT apple-ACC gave
b. Miki-ni Taro-ga/wa ringo-o ageta.
c. Ringo-o Taro-ga/wa Miki-ni ageta.
d. Taro-ga/wa ringo-o Miki-ni ageta.
'Taro gave Miki an apple.'

The different directionality of shifts between English and Japanese has puzzled many researchers because it ignores another strong factor of end-weight, which is accessibility, the tendency to place words that are easier to access earlier in the sentence. In fact, it is possible to study English end-weight without specifying a direction for distance minimization. Gries et al. (2018) conducted a corpus study of English genitive alternation. Weight difference is taken as the difference in length of characters between the possessor and the possessum. The syntactic role of the genitive phrase is left out of the picture, hence its direction of dependency with the verb too, for the subject and the object reside on different sides of the verb. Singaporean speakers and British speakers show different reaction to other factors but the short-before-long cue is difficult to override when the length-based preference becomes really strong. There is plenty of scope to explore this issue in the Japanese language with its strict verb-final order.

The performance theory as proposed by Hawkins (1994, 2004, 2014) can provide an elegant explanation of the superiority of the distance minimization account over the accessibility 
account. Other proposals include Chang (2009), which offers a connectionist model of syntax acquisition to explain the long-before-short preference in Japanese as an interaction between learning and processing. In other words, accessibility in a specific language can be a learned phenomenon. On top of this, the performance theory revolves around the central theme of a correspondence between performance and grammar which also explains aspects of word order typology (Hawkins 1990, 2014). The processing preference to minimize distance is termed the Minimize Domains (MiD) principle.

\section{(5) Minimize Domains (MiD)}

"The degree of this preference is proportional to the number of relations whose domains can be minimized in competing sequences or structures, and to the extent of the minimization difference in each domain." (Hawkins 2004; 31)

By considering the number of available competing sequences or structures, the MiD principle is able to attend to problems of word order biases in specific languages as well as in typological distributions.

This study will demonstrate two methods that are associated with the MiD principle. The first is the length difference as a predictor of performance data, as we have seen in the studies mentioned above. The second method is less used in the literature of weight effect, in which the distances to be minimized are calculated on the surface order, as if evaluating the processing efficiency after linearization. A case study with the Japanese adverb zitto 'motionlessly' will be presented to study the weight effect on its relative position with the direct object. Section 2 will first introduce general adverb behavior in Japanese. The difference between the two methods will be presented in Section 3, before Section 4 goes into details of the corpus study used to quantify weight effect. Results presented in Section 5 will lead to the discussion on missing pieces that have prevented weight effect from a wider application to studies of word order phenomena.

2. Adverb positions in Japanese. Japanese is a strict verb-final language with relatively free order on other constituents. It is a postpositional language with mandatory case marking following an accusative system. Adverbs are the third largest class after noun and verb, with 3071 types in the Balanced Corpus of Contemporary Written Japanese (BCCWJ), a hundred-millionword corpus (Maekawa et al. 2014). Adjectives are a smaller class, with about two thousand members, only one third of which can inflect to become an adverb, as in (6) and (9-d). The class of adverbs proper is mostly not analyzable back to other classes, except for a few with unreduced and less obscured lexicalized forms.

motigome o yawarakuku taki

mochi-rice ACC tenderly cook

'cook the mochi rice tenderly/till tender'

Adverbs have flexible positions in many languages. In Japanese, it is not surprising that the adverb can be placed even between the object and the verb, when the object can be displaced to an earlier position in the sentence for reasons of information structure and others. The position of Japanese adverb can be schematized in (7) and exemplified in (8).

(Adv) Subj (Adv) Obj (Adv) Verb. 
(8) Osoraku kyoo taro ga mata umaku kutusita o yawarakaku aratta.

Perhaps today Taro NOM again skilfully sock ACC tenderly washed

'Perhaps today Taro again skilfully and tenderly washed the socks.'

It is worth asking whether the position between the object and the verb requires special conditions for an adverb to occupy it, or even to be considered a canonical position for the adverb, which is not allowed in many other languages. Ultimately, we may even ask if the position in front of the verb is a conventionalized effect of end-weight acting on the object. If heavy objects only appear after the adverb and light objects can appear on either side, we may conclude that despite a general long-before-short preference, end-weight is still visible in Japanese when the constituent to be displaced does not have semantic dependency with the verb. If heavy objects only appear ahead of the adverb and light objects can appear on either side, it is likely that again the long-before-short preference overcomes end-weight.

Japanese adverbs may be subclassed in several ways. The one that is the most relevant to word order is the categorization by semantic functions: modal, time, manner, and resultative. Some example adverbs from the four subclasses are shown in (9) as used in Nanba \& Tamaoka (2016).

(9) a. Modal: ainiku 'unfortunately', osoraku 'perhaps', tabun 'probably'

b. Time: kyoo 'today', kinoo 'yesterday'

c. Manner: yukkuri 'slowly', kossori 'stealthily', sesseto 'industriously'

d. Resultative: barabara(ni) 'scattered', dorodoro(ni) 'in an ugly state', yawarakaku 'tender(ly)'

The four subclasses are shown to display different canonical orders by both online (Koizumi \& Tamaoka 2006) and offline methods (Nanba \& Tamaoka 2016). Koizumi \& Tamaoka (2006) observe that the four subclasses of adverbs demonstrate reading time difference in the constructions Adverb-Subject-Object-Verb (ASOV), SAOV, and SOAV, which may suggest surprisal (i.e. they run contrary to expectation). (10) shows the order of the reading time in each subclass. The smaller the reading time, the less surprisal has been generated by the construction.

$$
\begin{aligned}
& \text { a. Modal: ASOV }<\mathrm{SAOV}<\mathrm{SOAV} \\
& \text { b. Time: ASOV }=\mathrm{SAOV}<\mathrm{SOAV} \\
& \text { c. Manner and resultative: } \mathrm{ASOV}>\mathrm{SAOV}=\mathrm{SOAV}
\end{aligned}
$$

The scheme of Japanese adverb order may be represented as (11). The same scheme is echoed in the corpus study of Nanba \& Tamaoka (2016).

[MP (Modal-Adv) [IP (Time-Adv) Subj (Time-Adv) [vP (Manner/Resultative-Adv) Obj (Manner/Resultative-Adv) Verb ] Infl ] Modal ]

It must be noted that these studies did not control for the weight factor. They suggest that Japanese allows manner and resultative adverbs to be placed between the object and the verb, and yet there is still some way before claiming the order as canonical. Also, the categorization of adverbs into the subclasses is not straightforward. In fact, many resultative adverbs chosen in Koizumi \& Tamaoka (2006) are inflected adjectives, which may possess a tendency to be placed after the object by iconicity of change in state, which parallels the cases of valency patterns. Therefore, resultative adverbs are not considered in this study. 
3. Two methods to study weight effect. Weight effect is usually manifested by the shift in positions in the sentence for a constituent attaining a certain weight. We identify two explanations. The distance minimization account, or the Minimize Domains efficiency principle, is able to predict a short-before-long preference in English, a head-initial language, and a long-beforeshort preference in a head-final language like Japanese. The short-before-long preference in English may also be explained by the accessibility account which predicts a general preference in end-weight, which however is not evident in Japanese.

The length difference between two constituents to be ordered is a heuristic that can accommodate the predictions in either account, as studies show that it is the relative length of the constituents that matters (e.g. Hawkins 1994), though the studies may differ in the choice of length units, from character, to word or even bunsetu (a Japanese content word followed by a case marker or a postposition when applicable). A basic assumption is that the constituents in question retain their constituency. Cases such as (12) from Wasow (1997) will be excluded as "pictures of gruesome details of the victim's wounds" became discontinuous with the endweight. The same assumption applies to this study.

(12) a. The prosecution showed pictures of gruesome details of the victim's wounds to the jury.

b. The prosecution showed pictures to the jury of gruesome details of the victim's wounds

The length difference is a predictive method in nature, in the sense that the difference is calculated prior to the linearization process that may order the constituents in two (or more) distinct ways. While length difference can work for the MiD principle, the minimization preference came from an evaluation of the hypothetical ordering alternatives, which is the central theme of a performance-grammar correspondence in Hawkins' performance theory.

The difference between the two methods may be characterized relative to the timing of linearization for the same utterance, and is illustrated in Figure 1 with our case of object and adverb order in Japanese.

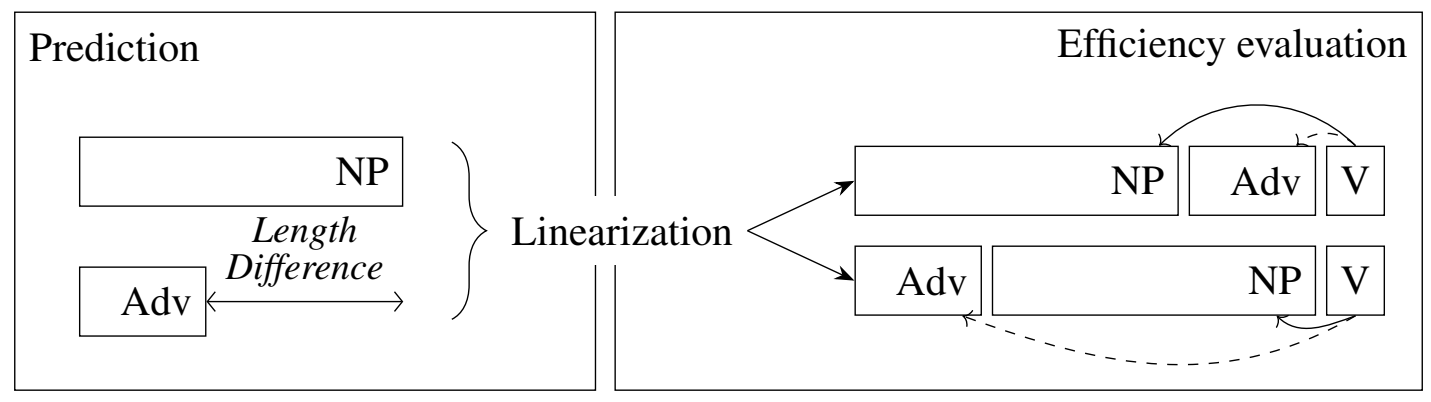

Figure 1. Two methods to formulate weight effect

With the method of efficiency evaluation, the MiD principle has to be understood with the concept of Phrasal Combination Domain (PCD), as described in (13).

Phrasal Combination Domain (PCD)

"The PCD for a mother node $\mathrm{M}$ and its $\mathrm{I}$ (mmediate) $\mathrm{C}$ (onstituent)s consists of the smallest string of terminal elements (plus all M-dominated non-terminals over the terminals) on the basis of which the processor can construct M and its ICs." (Hawkins 2004; 12). 
With either order in our case, the mother node is the VP that contains all three constituents of the verb (V), the accusatively marked object (NPo) and the adverb (Adv). In the adverbfirst order [Adv NPo V], the PCD stretches between Adv and V, and is the length of NPo. A Japanese sentence in this order is given in (14). The constituents on the borders of PCD are numbered with brackets. They will be left out in our calculation. The PCD is effectively the length of NPo counted by morae.

$$
\text { Setura wa zitto siroi kao o mitumeta. }
$$

Setura TOP motionlessly white face ACC stared-at.

PCD: $\quad(0) \quad 1-2-3 \quad 4-5 \quad 6 \quad$ (7)

'Setura motionlessly stared at the white face.'

In the object-first order [NPo Adv V], the PCD starts from the head of NPo and ends on V. A Japanese sentence in the object-first order is given in (15), with PCD counted by morae. Again, the constituents on the borders of PCD are left out in our calculation. The PCD is effectively the length of Adv.

Robin wa tikazuitekuru keeburu no nemoto o zitto
Robin TOP approaching cable GEN end ACC motionlessly observed
PCD:
'Robin observed the approaching cable end motionlessly.'

The preference for choosing the order [NPo Adv V] given a long NPo (i.e. a long-beforeshort order) is evaluated from a gain in efficiency by the minimization of PCD from that with [Adv NPo V] (PCD=length of NPo) to that with [NPo Adv V] (PCD=length of Adv). In other words, for a chosen order, for example [NPo Adv V], the gain in efficiency is the length of the closer constituent (Adv) subtracted from that of the farther constituent (NPo). The calculation will be presented with numeric data in (17) and (19).

Heuristically the gain in efficiency in terms of PCD is the same as length differences, leading back to the predictive method, but it is an essential step to comprehend the intention of the performance theory in explaining aspects of word order typology. A positive gain in efficiency means an instance in an efficient setting and a negative gain signifies a loss of efficiency. The harmonic orders of prepositions in verb-initial languages like English and postpositions in verb-final languages like Japanese can then be successfully derived.

4. Corpus study. This study will investigate the object and adverb order problem in Japanese with both the predictive method and the evaluative method, in the form of a corpus study as common with studies of weight effects (e.g. Hawkins 1994; Lohse et al. 2004; Bresnan et al. 2007). It allows us to investigate actual usage of heavy phrases. There is a significant amount of data available in the Balanced Corpus of Contemporary Written Japanese (BCCWJ) which contains more than a hundred million annotated words across different genres and registers (Maekawa et al. 2014). Constituency analysis is also available though one will need to tolerate a certain amount of errors.

The case study focuses on one adverb, zitto 'motionlessly', as a representative manner adverb of moderate frequency. A rough comparison in frequency with other adverbs is presented in Table 1. The situation of intervening adverbs between an object and a verb was estimated 
with the search template $[\mathrm{NPo} A d v \mathrm{~V}]^{1}$.

\begin{tabular}{lrrr}
\hline Adverb & Frequency & [NPo Adv V] \# & $\%$ \\
\hline zitto 'motionlessly' & 4238 & 937 & $22 \%$ \\
tira 'at a glance' & 1040 & 190 & $18 \%$ \\
tappuri 'in plenty' & 3563 & 598 & $17 \%$ \\
sikkari 'firmly' & 9800 & 1532 & $16 \%$ \\
zikkuri 'deliberately' & 1546 & 234 & $15 \%$ \\
kitinto 'accurately' & 6503 & 916 & $14 \%$ \\
\hline
\end{tabular}

Table 1. Frequency and percentage of [NPo Adv V] in BCCWJ

Adverbs of low frequencies can easily attain $0 \%$ or $100 \%$ of [NPo Adv V] without any implication of significance. Frequent adverbs are then defined by a frequency above 1000, which is arbitrarily picked. Table 1 shows the six adverbs with the highest percentage of the 'displaced' order [NPo Adv V]. This is a very rough estimation of the prominence of the intervened order as cases with more intervening constituents can easily escape from the template, but it is still noteworthy that zitto comes to the top of the list in percentage.

All 4238 instances of zitto in BCCWJ were manually analyzed for categorisation and weight effect analysis. Typical transitive constructions with zitto have been shown before but they are repeated here with lengths of the constituents in morae indicated below them. Length differences are measured by morae as phonological complexity, where $z i$ - $t$-to has three.

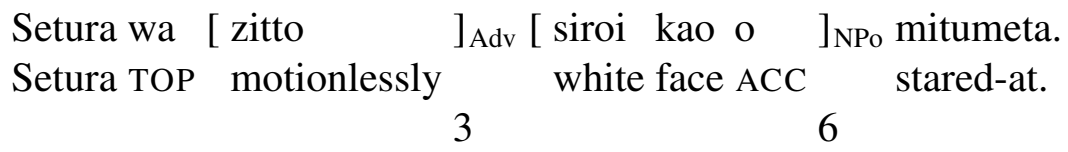

'Setura motionlessly stared at the white face.'

In (16), the object noun phrase is composed of an adjective, the head noun and the case marker. It has six morae. The predictive method will give the length of zitto subtracted by that of the noun phrase, that is, a length difference of three morae, as given in (17). Enclosing a constituent with vertical bars indicates its length. The evaluative method will work the other way round and give a length difference of minus three, indicating that this order is not so efficient.

a. Length difference in predictive method: $|\mathrm{NPo}|-|\mathrm{Adv}|=6-3=3$

b. Gain in efficiency in evaluative method: $|\operatorname{Adv}|-|\mathrm{NPo}|=3-6=-3$

In (18), the object is heavy, with 16 morae. Both predictive and evaluative methods give a length difference of 13, as shown in (19), and the order fulfils the long-before-short preference generally observed with Japanese.

\footnotetext{
${ }^{1}$ The actual search template was [NPo $\left.\operatorname{Adv}(-n i / t o) \mathrm{V}\right]$ with optional $n i$ or $t o$, which marks the instrumental case or quotatives respectively in other scenarios. They do not change the category or semantics of the adverb, though they may add subjective or objective reading (Martin 1988), yet again such reading is not absolute. There are voices for subjectivity-based explanations of adjective orders such as Scontras et al. (2017), but we contend that in the case of adverbs, subjective adverbs overlap largely with modal adverbs and objective adverbs with manner adverbs, and therefore subjectivity needs not be considered separately. NPo is identified by the proper noun class and the accusative case marker $o$.
} 
(18) Robin wa [ tikazuitekuru keeburu no nemoto o $]_{\text {NPo }}$ [zitto $]_{\text {Adv }}$ kansatusita. Robin TOP approaching cable GEN end ACC motionlessly observed $16 \quad 3$

'Robin observed the approaching cable end motionlessly.'

(19) a. Length difference in predictive method: $|\mathrm{NPo}|-|\mathrm{Adv}|=16-3=13$

b. Gain in efficiency in evaluative method: $|\mathrm{NPo}|-|\mathrm{Adv}|=16-3=13$

The heuristic formulas for weight effect under the two methods for both orders may be summarized in (20).
a. Length difference
[Adv NPo V] / [NPo Adv V]: $|\mathrm{NPo}|-|\mathrm{Adv}|$
b. Gain in efficiency
[Adv NPo V]: $|\mathrm{Adv}|-|\mathrm{NPo}|$
[NPo Adv V]: $|\mathrm{NPo}|-|\mathrm{Adv}|$

5. Results and discussion. All 4238 instances of zitto 'motionlessly' from BCCWJ were checked manually. 1790 instances match the template of [\{Adv NPo $\}$ V], where the order of Adv and NPo may switch. Without loss of generality, 76 cases are excluded for having extra intervening constituents. Other excluded cases include intransitive constructions and those when zitto is used as the predicate without a verb. Cases with an accusatively marked noun phrase are coded into the adverb-first order [Adv NPo V] or the object-first order [NPo Adv V]. The object-first order occupies $51 \%$, and the adverb-first order $49 \%$. We may say there is an equal preference for either order. This agrees superficially with the results of Koizumi \& Tamaoka (2006) and Nanba \& Tamaoka (2016).

5.1. LENGTH DIFFERENCE AS A PREDICTOR OF PERFORMANCE DATA. When we lay out the weight effect by the length difference between the object and the adverb, there seems to be a different picture. Length difference results are presented numerically in Table 2 . The percentages are calculated vertically, summing each column to $100 \%$. A number larger than $50 \%$ means that the order is preferred in the length difference range. Figure 2 directly plots the length of the object noun phrase since zitto has a fixed length and does not play a role in the distributions.

\begin{tabular}{lccccc|cccccc}
\hline & \multicolumn{1}{c}{ Adv $>$ NPo by } & \multirow{2}{*}{ NPo=Adv } & \multicolumn{7}{c}{ NPo $>$ Adv by } & \\
\cline { 2 - 7 } & $2+$ & 1 & & 1 & 2 & $3-4$ & $5-8$ & $9-16$ & $17-32$ & $33+$ & Total \\
\hline [Adv NPo V] & 5 & 86 & 213 & 232 & 104 & 135 & 89 & 12 & 0 & 0 & 876 \\
& $83 \%$ & $88 \%$ & $71 \%$ & $65 \%$ & $56 \%$ & $41 \%$ & $30 \%$ & $7 \%$ & $0 \%$ & $0 \%$ & \\
{$[$ [NPo Adv V] } & 1 & 12 & 87 & 125 & 82 & 191 & 207 & 157 & 49 & 3 & 914 \\
& $17 \%$ & $12 \%$ & $29 \%$ & $35 \%$ & $44 \%$ & $59 \%$ & $70 \%$ & $93 \%$ & $100 \%$ & $100 \%$ & \\
\hline Total & 6 & 98 & 300 & 357 & 186 & 326 & 296 & 169 & 49 & 3 & 1790 \\
\hline
\end{tabular}

Table 2. Length difference of NPo minus Adv

The maximum length of NPo went up to 44 morae in the object-first order but stopped at 17 in the adverb-first order. Weight effect is visible with a prominent long-before-short order when 
the object is 17 morae longer than zitto or more. The Minimize Domains principle is superior to the accessibility account as end-weight cannot be deduced from the results.

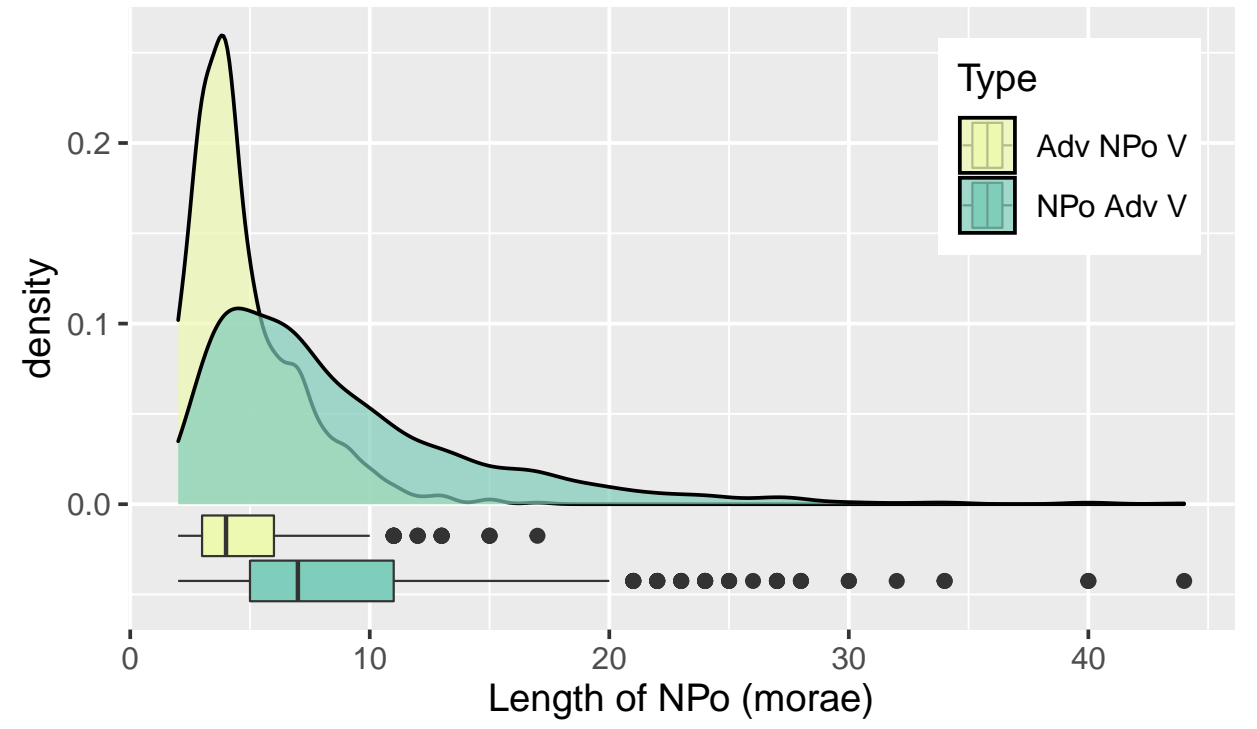

Figure 2. Length of the object noun phrase (NPo)

The predictive method challenges the canonicality of the object-first order, as we can see that when the difference in length between the object and the adverb is narrow, the adverb-first order is the dominating choice, until the difference reaches three morae. The vertical line in Table 2 divides the data into a preference for the adverb-first order and a preference for the object-first order. The crossing is also observable in Figure 2 with the different overlapping areas of the two distributions. The canonicality of the adverb-first order may be explained by a preference to place the object adjacent to the verb, which is a preferred position in many languages.

However, a difference of three morae is not a large number. It is possible that the preference becomes conventionalized at the point where the weight of the noun phrase grows past the marginal cases of a length difference of about two to three morae. By the performance theory, the object-first order may become the new normal. Furthermore, putting back about two thousand cases of intransitive sentences to the scene, zitto will show a prototypical position next to the verb. (21) shows a case where the object is topicalized and is absent in the verb phrase.

hyoomen no kagayaki wa zitto miteiru surface GEN radiance TOP motionlessly watching 'motionlessly watching the radiance of the surface'

If Japanese speakers have overcome the preference for the more accessible choice of endweight by conventionalization, one may imagine that three fourths of cases putting zitto adjacent to the verb would leave a stronger anticipation effect.

5.2. PCD AS AN EVAlUATOR OF PROCESSING EFFICIENCY. The evaluative method also uses the heuristic of length differences, but the subtraction formula is determined by the position of 
the constituents. Based on the concept of gain in efficiency on the Phrasal Combination Domain, the length difference is calculated by subtracting the length of the closer constituent from that of the farther constituent, as given in (20). Each order is considered separately as if examining typological variations so the results are also presented in separate tables.

Table 3 shows the length difference for the object-first order. The preference for processing efficiency is confirmed if an efficient order as defined by the MiD principle or the accessibility account dominates the distribution when the constituents grow in weight. ICL in the table denotes the longer immediate constituent, and ICS the shorter one.

\begin{tabular}{lccccc}
\hline $\mathrm{n}=914$ & $\mathrm{ICL}=\mathrm{ICS}$ & $\mathrm{ICL}>$ ICS by 1 & 2 & $3+$ & Total \% \\
\cline { 2 - 6 } ICL ICS V & \multirow{2}{*}{87} & $91 \%(125)$ & $99 \%(82)$ & $100 \%(607)$ & $89 \%$ \\
ICS ICL V & & $9 \%(12)$ & $1 \%(1)$ & $0 \%(0)$ & $1 \%$ \\
\hline
\end{tabular}

Table 3. Length of 1st constituent minus 2nd for the object-first order [NPo Adv V]

As expected from the well-observed long-before-short preference in Japanese, the object-first order is filled with instances that fulfil the expectation.

When we get to Table 4 for length difference with the adverb-first order, we see a different picture.

\begin{tabular}{lccccc}
\hline $\mathrm{n}=876$ & $\mathrm{ICL}=\mathrm{ICS}$ & $\mathrm{ICL}>\mathrm{ICS}$ by 1 & 2 & $3+$ & Total \% \\
\cline { 2 - 6 } ICL ICS V & 213 & $27 \%(86)$ & $5 \%(5)$ & $0 \%(0)$ & $10 \%$ \\
ICS ICL V & & $73 \%(232)$ & $95 \%(104)$ & $100 \%(236)$ & $65 \%$ \\
\hline
\end{tabular}

Table 4. Length of 1 st constituent minus 2nd for the adverb-first order [Adv NPo V]

With the same margin of three morae of length difference, the preference is flipped for a pattern that resembles an end-weight. If the MiD principle is responsible for the dominance of harmonic orders in word order typology, a language should be expected to abide by the same efficiency principle in its everyday usage.

However, there is still an obstacle before we can claim that the adverb-first order is under constant pressure under the MiD principle. Before weight effect comes into the picture, the adverb-first or object-adjacent order of [Adv NPo V] would be assumed to be the canonical order, as the object is the complement of the verb and the adverb only an adjunct. In other words, placing the object closer to the verb promotes certain efficiency. The logical conclusion will be to implement a complement-adjunct distinction into the MiD principle, as represented by the solid and dashed dependency lines on the right side of Figure 1.

5.3. TOWARD EFFICIENCY PROFILING WITH COMPLEMENT-ADJUNCT DISTINCTION. The predictive method of weight effect that has been widely adopted is backed by the Minimize Domains principle that started as an evaluative method of processing efficiency. The heuristic of length difference relies on an established ordering preference in the language. A longbefore-short or short-before-long preference will be too simplistic to address all the dynamics in performance data.

Results of the evaluative method on the zitto data are presented graphically in Figure 3. 


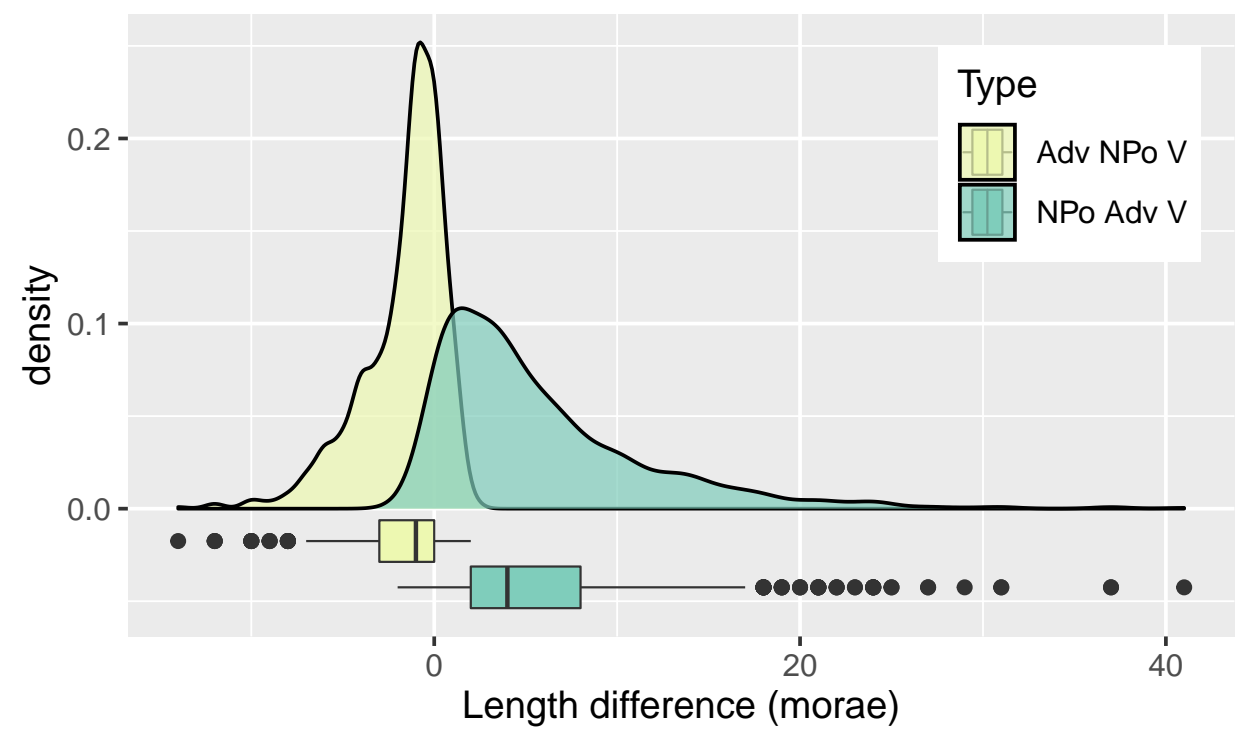

Figure 3. Gain in efficiency (length difference between 1st and 2nd constituents) as an efficiency profile

It represents the conformity of each instance to the efficiency principle. Collectively it can be understood as the efficiency profile of the competing constructions. The distribution for [Adv $\mathrm{NPo} \mathrm{V}]$ leans on the negative side, suggesting that it is under constant performance pressure, but this representation is flawed because it does not consider the gain in efficiency by placing the object closer to the verb. As we have been emphasizing, length difference is a heuristic to model weight effect with a formula that gives a single number. The results form an imaginary space of efficiency, two-dimensional in the current model. Like any heuristic, adding coefficients to the formula may help us see a different picture like in a duck-rabbit illusion. By applying different coefficients to the PCDs of object and adverb as a form of complementadjunct distinction, it is possible to shift the distributions toward a unified profile, implying that both orders are equally sensitive to weight effect and efficiency.

As an example, we may rework (16) into (22), where the length of the constituents in morae are indicated below them accompanied by arbitrary coefficients $c_{1}$ and $c_{2}$.

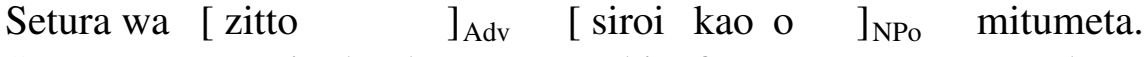

$$
\begin{aligned}
& \text { Setura TOP motionlessly white face ACC stared-at } \\
& 3 \times \mathrm{c}_{1} \quad 6 \times \mathrm{c}_{2}
\end{aligned}
$$

'Setura motionlessly stared at the white face.'

Under the current evaluative method, the gain in efficiency is negative given the short-beforelong order, and the number is $(3-6)=-3$. However, if we contend that the adverb may stretch from the verb twice as far away as an object without losing efficiency, we will set $c_{1}$ to be 2 and $c_{2}$ to be 1 . The calculation goes as $\left(3 \times c_{1}-6 \times c_{2}\right)=(3 \times 2-6 \times 1)=$ 0 , which is a neutral situation. In this way, we will be able to unify the efficiency profile of the adverb-first order with that of the object-first order, and state that both constructions are equally motivated in efficiency. 
6. Conclusion. This study is successful in pinpointing weight effect with a case study of the placement of the Japanese adverb zitto 'motionlessly' relative to the object. The results question existing studies that claimed the object-first order of [NPo Adv V] to be a canonical order alongside the adverb-first order of [Adv NPo V] because the weight factor has not been addressed. Two methods to formulate the weight effect, namely the predictive method and the evaluative method, have been demonstrated on the zitto data. While results from the predictive method tend to support the canonicality of the adverb-first order, the conclusion was superficial and lacking in explanatory power with performance data which derived the predictive method from the evaluative method by the Minimize Domains principle (Hawkins 1994) in the first place. On the other hand, the object-first order is preferred under the evaluative method, which evaluates the adverb-first order to be under constant performance pressure in the current calculation formula, because of the long constituent distance introduced by the heavy object in the middle, yet it gains processing efficiency with the shorter distance between the object and the verb. As a straight outcome after attending to both methods, the two orders showed overall equal preference in performance data, which is considered a new support to the MiD principle, by demonstrating how the efficiency principle operates at both levels of (1) the constituency construction of the full VP, which favors the object-first order, and (2) the Phrasal Combination Domain between the head of object and the verb, which favors the adverb-first order. It is proposed as a follow-up study to implement a complement-and-adjunct distinction in the MiD principle, as a step toward a more effective study method of weight effect which I shall call efficiency profiling.

\section{References}

Arnold, Jennifer E, Anthony Losongco, Thomas Wasow \& Ryan Ginstrom. 2000. Heaviness vs. newness: The effects of structural complexity and discourse status on constituent ordering. Language 76(1). 28-55. https://doi.org/10.1353/lan.2000.0045.

Bresnan, Joan, Anna Cueni, Tatiana Nikitina \& R. Harald Baayen. 2007. Predicting the dative alternation. In Gerlof Bouma, Irene Krämer \& Joost Zwarts (eds.), Cognitive foundations of interpretation, 69-94. Amsterdam: Royal Netherlands Academy of Arts and Sciences.

Chang, Franklin. 2009. Learning to order words: A connectionist model of heavy NP shift and accessibility effects in Japanese and English. Journal of Memory and Language 61(3). 374397. https://doi.org/10.1016/j.jml.2009.07.006.

Gries, Stefan Thomas, Tobias Bernaisch \& Benedikt Heller. 2018. A corpus-linguistic account of the history of the genitive alternation in Singapore English. In Sandra C. Deshors (ed.), Modeling World Englishes: Assessing the interplay of emancipation and globalization of ESL varieties, 245-280. Amsterdam: John Benjamins. https://doi.org/10.1075/veaw.g61.10gri.

Hawkins, John A. 1990. A parsing theory of word order universals. Linguistic inquiry 21(2). 223-261. https://www.jstor.org/stable/4178670.

Hawkins, John A. 1994. A performance theory of order and constituency. Cambridge: Cambridge University Press. https://doi.org/10.1017/CBO9780511554285.

Hawkins, John A. 2004. Efficiency and complexity in grammars. Oxford: Oxford University Press on Demand. https://doi.org/10.1093/acprof:oso/9780199252695.001.0001.

Hawkins, John A. 2014. Cross-linguistic variation and efficiency. Oxford: Oxford University Press. https://doi.org/10.1093/acprof:oso/9780199664993.001.0001.

Koizumi, M \& K Tamaoka. 2006. The canonical positions of adjuncts in the processing of Japanese sentences. Cognitive Studies 13(3). 392-403. https://doi.org/10.11225/jcss.13.392. 
Lohse, Barbara, John A. Hawkins \& Thomas Wasow. 2004. Domain minimization in English verb-particle constructions. Language 238-261. https://doi.org/10.1353/lan.2004.0089.

Maekawa, Kikuo, Makoto Yamazaki, Toshinobu Ogiso, Takehiko Maruyama, Hideki Ogura, Wakako Kashino, Hanae Koiso, Masaya Yamaguchi, Makiro Tanaka \& Yasuharu Den. 2014. Balanced corpus of contemporary written Japanese. Language Resources and Evaluation 48(2). 345-371. https://doi.org/10.1007/s10579-013-9261-0.

Martin, Samuel E. 1988. A reference grammar of Japanese. Clarendon, VT: Tuttle Publishing. https://doi.org/10.1515/9780824843861.

Nanba, Emi \& Katsuo Tamaoka. 2016. A corpus-based investigation of the canonical order of manner and resultative adverbs. Gengo Kenkyu 150. 173-181.

Ross, John Robert. 1967. Constraints on variables in syntax. Cambridge, MA: MIT dissertation.

Scontras, Gregory, Judith Degen \& Noah D. Goodman. 2017. Subjectivity predicts adjective ordering preferences. Open Mind 1(1). 53-66. https://doi.org/10.1162/OPMI a 00005.

Wasow, Thomas. 1997. Remarks on grammatical weight. Language Variation and Change 9(1). 81-105. https://doi.org/10.1017/S0954394500001800.

Yamashita, Hiroko \& Franklin Chang. 2001. "Long before short" preference in the production of a head-final language. Cognition 81(2). B45-B55. https://doi.org/10.1016/s0010-0277(01)00121-4. 